Santé mentale au Québec

Analyse d'une intervention clinique en fonction des outils élaborés par l'approche ethnopsychiatrique de Devereux et Nathan Analysis of a clinical intervention according to instruments inspired by the ethnopsychiatric approach elaborated by Devereux and Nathan

Análisis de una intervención clínica en relación con los instrumentos elaborados por el enfoque etnopsiquiátrico de Devereux y Nathan.

Análise de uma intervenção clínica em função das ferramentas elaboradas pela abordagem etnopsiquiátrica de Devereux e Nathan

Marie-Rosaire Tshisekedi-Kalanga et Gisèle Legault

Volume 30, numéro 2, automne 2005

Le soutien social

URI : https://id.erudit.org/iderudit/012147ar

DOI : https://doi.org/10.7202/012147ar

Aller au sommaire du numéro

\section{Éditeur(s)}

Revue Santé mentale au Québec

ISSN

0383-6320 (imprimé)

1708-3923 (numérique)

Découvrir la revue

Citer cet article

Tshisekedi-Kalanga, M.-R. \& Legault, G. (2005). Analyse d'une intervention clinique en fonction des outils élaborés par l'approche ethnopsychiatrique de Devereux et Nathan. Santé mentale au Québec, 30(2), 233-255.

https://doi.org/10.7202/012147ar

\section{Résumé de l'article}

Cet article analyse une intervention clinique effectuée au Module d'ethnopsychiatrie de l'Hôpital Jean-Talon de Montréal. Les auteurs présentent d'abord un rappel de certaines notions centrales d'une approche ethnopsychiatrique developpée en France par Nathan en réponse aux problèmes de santé mentale vécus par les migrants. La présentation de l'intervention des auteurs s'inscrit dans la trajectoire de cette approche qui est en processus d'élaboration et d'expérimentation au Québec. La situation clinique est ensuite présentée suivie de l'intervention effectuée par le Module d'ethnopsychiatrie. Enfin, les auteurs terminent avec une analyse de la situation sur le plan psychosocial, psychodynamique (étiologie moderne) et culturel (étiologie traditionnelle). 


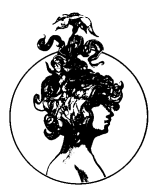

\title{
Analyse d'une intervention clinique en fonction des outils élaborés par l'approche ethnopsychiatrique de Devereux et Nathan
}

\author{
Marie-Rosaire Tshisekedi-Kalanga* \\ Gisèle Legault ${ }^{\star \star}$
}

\begin{abstract}
Cet article analyse une intervention clinique effectuée au Module d'ethnopsychiatrie de l'Hôpital Jean-Talon de Montréal. Les auteurs présentent d'abord un rappel de certaines notions centrales d'une approche ethnopsychiatrique developpée en France par Nathan en réponse aux problèmes de santé mentale vécus par les migrants. La présentation de l'intervention des auteurs s'inscrit dans la trajectoire de cette approche qui est en processus d'élaboration et d'expérimentation au Québec. La situation clinique est ensuite présentée suivie de l'intervention effectuée par le Module d'ethnopsychiatrie. Enfin, les auteurs terminent avec une analyse de la situation sur le plan psychosocial, psychodynamique (étiologie moderne) et culturel (étiologie traditionnelle).
\end{abstract}

epuis plus d'un siècle, les pays occidentaux accueillent bon nombre
de personnes qui immigrent par choix ou par contrainte. Il se pose alors des problèmes d'adaptation et d'intégration de tous ordres tant pour le migrant que pour la société d'accueil. Plusieurs de ces problèmes sont pris en charge par les intervenants sociaux. Sur le plan de la santé mentale toutefois, les cliniciens sont confrontés à des situations complexes lorsqu'il s'agit de personnes issues de sociétés non occidentales et dont les propos sont significativement marqués par des processus de codage culturel. L'intérêt pour des modes de soins appropriés à ces clientèles remonte à Kraepelin au début du siècle (Kirmayer et Minas, 2000), plusieurs lui ont succédé principalement au cours des années soixante-dix où les contributions de Kleinman (1977) et Devereux $(1970,1972)$ ont alimenté la réflexion sur les bases théoriques et la pratique psychiatrique en tant que productions culturelles qu'il fallait interroger. Dans la suite de leurs travaux, certaines cliniques occidentales ont tenté d'adapter leurs approches à la réalité de ces migrants

École de service social, Université de Montréal.

** Clinique externe de psychiatrie, Hôpital Jean-Talon. 
quoique le nombre de centres thérapeutiques spécialisés dans leur traitement soit limité. En Europe, le Centre de Santé Mentale C. W. Laeken de Bruxelles, le Centre Minkowska de Paris et le Nafsiyat Intercultural Therapy Center de Londres font partie de ces centres. A Montréal, le «cultural consultation service», affilié à l'université McGill et dirigé par le Dr. L. Kirmayer s'inscrit dans cette même perspective.

En 1979, en France, Nathan (1986, 1988, 1994), suivant les pas de Devereux, a réussi à mettre sur pied une clinique de consultation qui tente de tenir compte des dimensions culturelles et religieuses du discours des patients; il a ainsi élaboré une approche basée sur certains apports de la psychanalyse et de l'anthropologie. De par ses dispositifs techniques et ses stratégies thérapeutiques, son approche représente une thérapie qui tente d'être plus interculturelle. L'Hôpital Jean-Talon l'utilise depuis maintenant dix ans. Notre choix s'explique par le fait que plusieurs des membres de notre Module d'Ethnopsychiatrie ont fait des séjours en France et ont été exposés aux enseignements et méthodes de travail des thérapeutes exerçant dans ce pays.

Nous présentons dans un premier temps, certaines notions importantes et centrales de l'approche de Devereux et Nathan pour ensuite analyser leur application éventuelle à une situation clinique précise. Nous espérons ainsi démontrer que cette approche permet une mobilisation des pensées et des affects par la prise en compte des éléments personnels, culturels, historiques et politiques du patient et de son groupe familial.

\section{Notions de base de la théorie ethnopsychiatrique de Devereux et Nathan}

\section{Le complémentarisme}

Le complémentarisme, tel que développé entre autres par Devereux (1972), part du principe que certains phénomènes humains ne relèvent pas de la psychanalyse ou de l'anthropologie exclusivement mais plutôt d'un double discours qui se veut à la fois obligatoire et non simultané, ces deux discours sont alors dits complémentaires. Ainsi les éclairages successifs de la psychanalyse et de l'anthropologie sont utilisés pour comprendre les situations à l'étude et intervenir. A cet effet, la prise en compte des variables psychodynamiques et culturelles dans une approche thérapeutique offre un espace à ces deux domaines de connaissances dans une complémentarité féconde. La méthodologie clinique de Devereux et Nathan reflète ce double emprunt. 
"C'est précisément la possibilité d'expliquer "complètement" un phénomène humain d'au moins deux manières (complémentaires) qui démontre, d'une part, que le phénomène en question est à la fois réel et explicable et, d'autre part, que chacune de ces deux explications est "complète" (et donc valable) dans son propre cadre de référence. Bref, le "double discours" rend possible une double prévisibilité du phénomène, car chacun des deux moyens de le prédire le rend inévitable dans le cadre du système explicatif que l'on utilise.» (Devereux 1972, 9).

\section{Les théories étiologiques traditionnelles}

Les théories étiologiques traditionnelles sont à mettre en tandem avec les théories étiologiques «scientifiques» modernes car «l'expérience clinique suggère que les patients provenant de cultures non occidentales ne peuvent dérouler leurs associations d'idées que dans le cadre de théories étiologiques dites traditionnelles » (Streit, 1996). Ces théories tendent à indiquer les causes et surtout le sens des maladies. «Nathan précise que le terme étiologie n'a pas la même portée que dans la pensée médicale occidentale, car les étiologies traditionnelles sont interrogatives, multiples et non exclusives; elles inaugurent le discours » (Streit, 1996, 389). Dans leurs théories étiologiques, les grands systèmes de thérapies traditionnelles, telle que la possession, le chamanisme, la voyance, accordent à certains objets une fonction explicite et ineffable permettant d'amener le consultant, durant le processus thérapeutique, à donner un sens aux perturbations du patient afin de l'aider à livrer ses angoisses et à ainsi retrouver un état de soulagement, de sécurité et de protection.

À cet effet, les considérations de Laplantine $(1988,38)$ sont éclairantes: "Si l'on considère la gamme la plus étendue des sociétés, force nous est de reconnaître que les représentations étiologiques incriminées sont davantage de nature exogène (possession par un esprit pathogène, agression par un sorcier, infection par un virus) que de nature endogène (c'est dans la constitution de l'individu comme en homéopathie, dans son patrimoine comme en génétique, ou encore dans ses propres conflits psychologiques, qu'il faut rechercher la formation de ses troubles). Et corrélativement la majorité des "psychiatries populaires" pensent que les facteurs de la guérison sont davantage de nature exogène (suggestion, drogue, extirpation symbolique du mal souvent matérialisée par un objet) qu'endogène (tels les rêves en des sanctuaires comme au Maroc, ou les associations libres en psychanalyse par le moyen du transfert).» 


\section{Les notions de soi et de personne}

Nous évoquons brièvement ici les notions de soi et de personne telles qu'explicitées par les modèles collectif-communautaire et individualiste-égalitaire de la personne introduits par Cohen-Emerique (1990). Les patients migrants, dans leur très grande majorité, viennent de sociétés «holistes» ou à modèle collectif-communautaire et sont alors confrontés à des modèles d'intervention s'inspirant d'un modèle individualiste de la personne en contradiction avec leur conception d'une personne étroitement intégrée dans son univers social, d'où la nécessité de développer d'autres approches qui en tiennent compte telle l'ethnopsychiatrie.

Dans ses écrits sur ces modèles, Cohen-Emerique réfère aux travaux de Dumont (1978) qui, dans ses recherches sur la conception moderne de l'individu, dresse un tableau des visions «holistes» et modernes de la personne. Dans le modèle collectif-communautaire, modèle auquel se référent la majorité des nouveaux arrivants, l'autre est inclus dans la définition de la personne, cette dernière est perçue comme un être fondamentalement social qui exprime ses qualités ou son unicité à travers un engagement mature à sa famille et à son groupe social (Kirmayer 1989). Dans les sociétés à dominante collectiviste-communautaire, les liens entre les individus sont étroits et forts, chacun a un puissant sentiment d'appartenance à sa famille et à sa communauté d'origine. Le nous prédomine sur le soi. Il y existe une sorte d'échange entre l'allégeance des individus à la famille, au groupe, au village, à la tribu et la contrepartie protection fournie en retour. La patience, la coopération et la conciliation sont encouragées.

Le concept de la personne du modèle individualiste-égalitaire est d'autre part centré sur l'individualisme, être une personne égale être un individu. Chaque personne est dite unique et devant poursuivre ses buts personnels. Les personnes sont valorisées selon le degré de développement et d'articulation de leur moi (self) et leur capacité d'autodirection (Kirmayer 1989), l'autonomie et l'indépendance de la personne sont valorisées impliquant une séparation physique et morale de sa famille à l'âge adulte. Le soi est privilégié plutôt que l'appartenance à la famille ou au groupe. Dans ce modèle chacun veille sur ses intérêts propres et une très grande liberté est laissée aux membres des sociétés issues de ce modèle. Les réalisations de soi sont mesurées en terme de possessions matérielles ou de contrôle de l'environnement. 


\section{Initiation, filiation et affiliation}

Dans toutes les cultures, la vie est scandée par certains rythmes et rites qui accompagnent les passages d'un état, d'un statut social à un autre: ainsi la naissance, le sevrage, la première coupe de cheveu, la circoncision, la puberté, le mariage, le premier enfant, etc. L'idée derrière ces rites et cérémonies de passage est de ne pas laisser la personne se métamorphoser toute seule, de l'accompagner. Dans les cultures où la personne est perçue en termes de relations sociales et d'appartenances, il va de soi que le groupe (ou le groupe initiatique restreint) l'accompagne nécessairement lors de ces passages. La personne qui consulte parce qu'elle est en état de déséquilibre, se retrouve seule, fermée, isolée, en besoin. Donc, ces rituels initiatiques l'affilieront de nouveau à son groupe d'appartenance, l'introduisant dans le groupe des être visibles et invisibles de sa culture. Le processus initiatique débouche donc sur l'affiliation de la personne en difficulté à son groupe, le groupe «entrera» en quelque sorte dans la personne de façon à ce qu'elle ne soit pas une subjectivité close. Cette initiation est difficile et plus rare dans les sociétés où le modèle individualiste domine car le sentiment d'appartenance est faible sinon inexistant.

L'initiation par excellence demeure toutefois la naissance où l'enfant est considéré comme «entre deux mondes » pendant un certain temps, il est un voyageur fragile, peut-être revient-il (réincarnation d'un ancêtre), restera-t-il, s'en ira-t-il ? On y va donc doucement avec lui et on attend quelques jours pour le nommer. Il faut d'abord identifier qui est cet enfant, sa nature, le découvrir avant de pouvoir le nommer. Ce processus est l'affaire de la famille élargie et même souvent de toute la communauté et non la seule responsabilité des parents. Ainsi on consultera souvent un initié qui s'enquerra des rêves, des événements de la grossesse, du contexte de la naissance, on scrutera le placenta quelquefois. Il est ainsi très important de bien nommer l'enfant «c'està-dire d'identifier le plus tôt possible sa véritable nature car un nom maladapté peut le rendre malade, pire encore, l'inciter à repartir, c'està-dire à mourir » (Moro 1992, 90).

Connexe à l'initiation, apparaît la notion de protection dont il faut se garantir afin d'effectuer les passages avec succès. Rituels et objets de protection sont donc nécessaires lors des passages; à cet effet, l'accouchement en pays d'accueil est un passage particulièrement délicat. Cet accouchement se fait sans l'aide du groupe des femmes qui entourent habituellement l'accouchée et lui sert en quelque sorte de matrice, la femme accouche donc "dans un monde de blouses blanches », hostile, froid qui ne lui est pas coutumier, des perturbations 
mère-enfant peuvent s'ensuivre ou se révéler plus tard au cours des premières années de l'enfant. Lorsque la notion de protection est évoquée et que le groupe thérapeutique porte la mère elle-même, elle peut de nouveau porter, protéger, éduquer son enfant.

\section{Le groupe comme dispositif}

«Nathan (1991) souligne qu'une approche groupale s'avère cruciale et représente l'un des dispositifs principaux de l'approche ethnopsychanalytique. La conception de la personne (ajout) et le fonctionnement des systèmes étiologiques traditionnels exigent un dispositif groupal, car ces systèmes qui comportent une médiation entre des univers (par exemple, ordinaire et extraordinaire) ne peuvent fonctionner dans une situation duelle. Le groupe qui reçoit le patient ainsi que les membres de sa famille est composé d'une quinzaine de thérapeutes, c'est-à-dire de professionnels (psychologues, psychiatres et médecins) ayant souvent une formation psychanalytique. Ces thérapeutes sont d'origines culturelles diverses et assurent conséquemment des lectures diverses d'une même réalité. » (Streit 1996, 388).

Les choses doivent être dites en groupe car ce groupe rappelle au migrant que, chez lui, l'individu ne consulte pas dans une situation de face-à-face mais le plus souvent dans un contexte groupal. L'éthique de la confidentialité devient l'éthique du témoin (du visage de l'autre), les choses sont dites devant témoins (visibles et invisibles dont l'ancêtre est souvent le premier évoqué de ces invisibles), ce qui amène détente et appui, appui du retour à la culture d'origine, base de l'humanité de la personne. Le patient migrant est alors arraché à l'universalité et placé dans ses appartenances, ce qui le rend profondément humain.

Selon Nathan (1991), le groupe assure plusieurs fonctions :

- une fonction d'étayage ou de portage: il s'agit d'un portage culturel (on parle la langue maternelle du patient et on fait référence aux étiologies traditionnelles) et d'un portage psychologique (le patient peut s'appuyer sur le groupe);

- une fonction de médiation entre les thérapies traditionnelles qui se déroulent principalement en groupe et la thérapie psychanalytique (on estime souvent, dans les sociétés non occidentales, que la maladie concerne le groupe familial en plus de l'individu malade et, par conséquent, les approches thérapeutiques sont généralement groupales);

- une fonction de médiation de la relation entre le patient et le thérapeute principal (les cothérapeutes, tout particulièrement 
ceux partageant la même origine ethnique que le patient, facilitent l'évocation des étiologies traditionnelles et, de ce fait, l'accès à la vie intime de sujet).

Comme il s'agit d'un groupe multiculturel, les étiologies traditionnelles énoncées au cours d'une séance sont acceptables et paraissent même naturelles (Streit 1996, 388).

Le bombardement sémantique est un processus actif de compréhension d'un fragment de la problématique du patient à travers le regard de plusieurs cultures. Il offre au patient plusieurs visions de ce qu'il vit, tout en respectant ses limites de compréhension de sa propre culture. A la fin de ce travail, il se voit présenter une réorganisation de ses propos à partir des «inputs» du groupe constitué de plusieurs cothérapeutes de cultures et de professions diverses offrant autant de lectures de sa réalité problématique (Nathan, 1988).

\section{Présentation d'un cas}

Nous présentons ici un cas où nous sommes à même de reconnaître l'utilisation de ces notions dans une prise en compte des divers éléments à la fois personnels, familiaux, culturels et politiques de la situation.

La demande de consultation a été adressée au module d'ethnopsychiatrie de l'Hôpital Jean-Talon par la travailleuse sociale d'un CLSC de l'Ouest de Montréal. Le but de la demande est de venir en aide à la famille Costa1 qui, dépassée par le comportement de leur fils cadet Michel, âgé de 24 ans, sollicite une aide psychologique. La famille le trouve déprimé, explosif et en opposition farouche à sa mère qui le décrit comme rebelle. L'élément précipitant remonte au temps des fêtes de l'année précédente où Michel, sous l'effet de l'alcool, a fracassé le visage de son cousin lors d'une bagarre. La demande de consultation s'est faite avec empressement par la mère au nom de la famille et en accord avec Michel. Pour faciliter la compréhension des événements, la travailleuse sociale a pris soin de présenter les grands moments de l'histoire migratoire de la famille.

\section{Histoire de Michel et sa famille}

Michel est un jeune homme célibataire. Il est né au Québec de père uruguayen et de mère argentine. Il s'exprime parfaitement en français et en espagnol. Il est chrétien de la branche des mormons ${ }^{2}$ et pratique régulièrement sa religion. Il n'a pas terminé ses études au secondaire (secondaire III non complété), il a aussi suivi une formation professionnelle de soudeur qu'il n'a pas terminée. Il a travaillé comme gardien de sécurité, puis a fait plusieurs petits emplois. Actuellement, il ne 
travaille pas et est bénéficiaire de l'aide sociale, il vit sous le toit familial.

Madame Costa, âgée aujourd'hui de 50 ans, est originaire d'Argentine et de descendance indienne, elle est de confession protestante. Dans son pays, elle travaillait comme coiffeuse. En 1973, elle rencontre, en Argentine, Monsieur Costa, originaire d'Uruguay, qui y faisait des activités de commerce. Ce dernier est issu d'une famille modeste mais éduqué dans le luxe par ses parents qui étaient gardiens d'une villa de gros propriétaires terriens. Il est chrétien de la branche des mormons dont il est un des leaders. La famille de madame se serait opposée à leur union pour des raisons qui semblent secrètes. Un an après leur mariage, le couple immigre au Canada et s'installe au Québec suite à l'instabilité politique de l'Argentine. En 1976, le couple a un premier enfant qu'ils prénomment Serge. L'accouchement se fait par césarienne; le père n'est pas content de l'arrivée de cet enfant. Onze mois plus tard, c'est la naissance de Michel, l'accouchement se fait aussi par césarienne. Les circonstances de la naissance de ce deuxième enfant sont pénibles et madame fait une dépression à la suite de cette grossesse de nouveau non désirée par le père. Madame précise n'avoir pas connu le bonheur d'une grossesse car elle était triste et troublée à chaque fois. À la naissance de Michel, madame pressentait qu'elle allait être seule à l'aimer, Monsieur aurait toutefois fini par aimer ses enfants selon ses propos.

Par la suite, le couple connaît des difficultés financières et madame est contrainte de travailler dans une manufacture tout en suivant des cours de francisation au COFI (centre d'Orientation et de Formation des immigrants). Pendant ce temps, monsieur tient un garage où il trafique des «transactions louches» selon madame. En 1982, monsieur fait faillite et la famille décide de quitter le Québec pour l'Uruguay. Michel a alors cinq ans. Il dit avoir de très bons souvenirs de son enfance au Québec. Ses années d'école primaire en Uruguay ont été difficiles sur le plan de l'intégration sociale et scolaire compte tenu qu'il ne parlait pas la langue du pays. Avec le temps, Michel a toutefois fini par trouver sa place et à se faire des amis. Il décrit toutefois cette période de huit ans de vie en Uruguay comme difficile au point de vue relationnel avec son père. Il se rebellait contre lui car il le trouvait trop sévère à son égard. Il craignait ses réactions car il le punissait pour un oui ou pour un non. Il s'est alors beaucoup rapproché de sa mère.

La famille revient au Québec en 1994, Michel a alors treize ans. Selon sa mère, Michel était très déprimé et troublé de se sentir déraciné à la suite de ce retour; en vue de son intégration sociale et scolaire, Michel a dû réapprendre le français qu'il ne parlait plus. Il a alors été 
placé dans une classe d'accueil et ses résultats scolaires ont été faibles. Il s'est toutefois fait un réseau social qu'il a dû laisser en 1995 lorsque sa famille a quitté une nouvelle fois le Québec pour l'Argentine. Cette même année, Michel vit le divorce de ses parents. Le père de Michel quitte alors l'Argentine pour aller vivre en Uruguay, son pays d'origine, alors que madame y demeure avec les deux garçons. À 18 ans donc, il décide de suivre son père et de ramener un peu d'argent à sa mère et son frère qui vivaient alors pauvrement en Argentine. Michel décrit cette période auprès de son père, comme la plus noire de son existence. Il a été laissé à lui-même et a connu la misère, la faim, la privation, le désespoir. Il a aussi consommé de l'alcool et de la drogue. Il a été malheureux de voir son père dilapider tout l'argent de la vente de la maison familiale, il s'est alors enfermé pendant un mois dans une chambre mal éclairée où il lisait la Bible. Il décide ensuite de quitter son père et de retourner en Argentine auprès de sa mère et de son frère.

En 1996, Michel retourne au Québec avec sa mère et son frère Serge. Il socialise en reprenant contact avec les personnes qu'il avait connues auparavant et consomme également beaucoup de drogue et d'alcool. En 1998, Motus, le conjoint chilien de sa mère, entre dans la vie de la famille. Le rapport Michel-Motus ne semble pas très chaleureux.

Au moment de la consultation, madame travaille comme aide familiale dans une coopérative de soutien à domicile. Serge le fils aîné, est travailleur autonome et très impliqué dans les arts martiaux. Michel est actuellement à la recherche d'un emploi. À la maison, il passe son temps à regarder la télévision ou des vidéos.

La famille aura donc connu en tout cinq migrations, c'est-à-dire : Argentine-Québec, Québec-Uruguay, Uruguay-Québec, QuébecArgentine et Argentine-Québec. En d'autres mots, en l'espace de quinze ans, Michel et son frère auront vécu quatre changements importants de lieu de résidence.

\section{Avis professionnel et recommandations de l'Équipe}

Étant donné les multiples migrations de cette famille et l'instabilité du fonctionnement familial à tous les niveaux de même qu'à la personnalité du père, on estime que Michel n'a pu traverser les phases de sa croissance et qu'il en est sérieusement perturbé. L'équipe d'intervention estime que Michel est en train de développer une forme de psychose, sa souffrance de même que celle de sa famille et leur périple migratoire demandent à être pris en charge et portés par un groupe de thérapeutes de différentes cultures. 


\section{Intervention du Module d'ethnopsychiatrie}

Le groupe se prépare à recevoir la famille: il est composé d'une thérapeute principale (TP), criminologue d'origine québécoise et dix cothérapeutes (CT) de cultures et professions diverses dont deux sont de la même aire culturelle que la famille (voir graphique). L'un d'eux agira comme médiateur culturel.

Lors de la première rencontre avec la famille, le groupe privilégie certaines pistes telles connaître le motif de la rencontre selon les concernés, connaître leur histoire à travers leur récit migratoire, leurs liens familiaux au pays d'origine et au Québec.

La famille est très motivée face à la prise en charge proposée par le Module, une formule qui leur offre un espace où tous les membres peuvent s'exprimer dans leur langue maternelle. Ils espèrent une amélioration de la situation de Michel et son intégration personnelle et sociale. Cinq rencontres ont eu lieu en l'espace de six mois avec cette famille qui a démontré sa détermination à poursuivre la démarche et désirant en faire bénéficier au plus grand nombre possible des membres de leur famille.

À la première rencontre, la mère, Motus et Michel sont présents. On y refait l'histoire de la famille et la mère parle de la sensibilité de son fils Michel aux divers événements survenus dans la famille. Elle précise que c'est vers l'âge de huit ans que son fils a changé et qu'il a été plus souvent de mauvaise humeur. Aujourd'hui, tout lui est une montagne et il semble connaître plusieurs malheurs. Michel, quant à lui, évoque la période où il était au Québec comme un moment de bonheur. Il insiste sur son appartenance québécoise. Il se décrit comme rebelle, n'a plus confiance en lui et tout ce qu'il entreprend est gauche au point que cela provoque des disputes entre lui et sa mère. Le divorce de ses parents l'a beaucoup affecté et le lien avec son père est présentement coupé malgré lui. La famille fournit un matériel clinique très riche au fur et à mesure que le travail thérapeutique avance. De cette rencontre, le groupe retient la fragilité de Michel et de sa famille de même que l'instabilité presque permanente de leur parcours migratoire. Ce dernier a beaucoup affecté Michel dans sa formation identitaire et a fomenté une colère perceptible lors de la rencontre.

Ainsi à la fin de la première rencontre, la thérapeute principale donne comme prescription ${ }^{3}$ à Michel d'apporter quelques objets des différents endroits où il a vécu afin d'essayer de relier tous ces mondes morcelés. Elle recourt alors à la légende du fil d'Ariane ${ }^{4}$ coupé plusieurs fois et qu'il faut rattacher et raccorder. 
À la rencontre suivante, Michel et sa mère sont présents. Michel apporte un objet de chaque pays où il a vécu - entre autres un chandail d'Argentine et un beau sac en cuir d'Uruguay qui sert à transporter le «maté ${ }^{5}$ » et quelques films de son enfance au Québec. Sur le sac portematé de Michel, figure le portrait de Che Guevara. Il s'agit d'un cadeau reçu en Uruguay d'un oncle vivant à la campagne qui possède des chevaux et qui le trouvait sympathique. À cette époque, Michel avait entre 10 et 11 ans. Il connaît bien l'histoire du Che, qui est pour lui, un modèle.

La thérapeute principale fait le choix de se servir du Che dans l'entrevue comme modèle masculin afin de renforcer Michel et de raviver ses forces positives. Michel apparaît ici en besoin d'affiliation, en mal d'identification masculine à un père absent et en raison de plusieurs appartenances culturelles disparates. Aucun des groupes culturels auxquels il a appartenu n'a pu le traverser et l'aider à se constituer en entité consistante. À la fin de l'entrevue, Michel pose des questions en rapport avec la folie et son intégrité personnelle. Ces questions semblent être en lien avec l'anxiété de séparation soit par rapport à sa famille soit par rapport au groupe. Michel semble se sentir comme l'anormal, le fou, le malade qui perturbe les autres et qu'on pourrait rejeter.

À la troisième rencontre, toute la famille est là, c'est-à-dire, la mère, Serge, Motus et Michel qui dit aller beaucoup mieux et avoir moins de problèmes. Il dit aussi : «je commence à devenir mature». Par contre, Serge parle de sa préoccupation concernant son frère en ramenant l'histoire de la «Barra Blanca», dont nous donnons un extrait. Nous notons que la mère, Motus et Michel lui-même avaient occulté jusque là cette histoire.

\section{Extrait 1:}

TP: (s'adressant à Serge). Ton point de vue sur Michel?

Serge: J'ai perdu la patience de parler avec lui, il va sortir sa théorie. Je me frustre, il s'enferme dans un mur et je ne peux pas le percer, il répond des choses complètement incohérentes. Il lui est arrivé quelque chose qui l'a choqué. J'ai un doute. En Argentine, il était à la plage et avait bu beaucoup d'alcool. Il y avait là un groupe de 50 personnes, des fans de soccer "La Barra Blanca» réputés pour être violents. Un ami de Michel s'est approché du groupe et l'a présenté au chef de la clique qui lui dit: Veux-tu connaître Satan? » Michel dit non, je suis un fils de Jésus. Michel et son ami ont été battus. Mais je sais jusqu'où ils peuvent aller, battre, même violer. C'est une secte satanique. On va ensuite chez des amis, on boit, et sur le lit, Michel fait 
deux fois de suite une crise "aidez-moi, ils sont en train de m'enculer, le diable est en train de m'enculer»

Michel: Le gars, c'était un fou... il disait: Est-ce que tu veux te marier avec Satan? Devant une station d'essence, il a bu de l'essence, il était très fou. Je savais qu'il n'était pas Satan.

Cette histoire étrange a permis au groupe de saisir l'ampleur des difficultés de Michel et l'importance du traumatisme qu'il a alors vécu. Toujours selon Serge, son frère a regardé plus de quarante fois le film «Le Corbeau », réputé satanique. Chaque fois Michel change et devient bizarre. Il pourrait alors s'agir, pour Michel, d'une tentative de maîtriser le traumatisme par un visionnement répétitif du film.

Pour l'équipe de travail, la rencontre a été marquée par les précisions apportées par Serge au sujet de son frère et aussi par l'information sur les pratiques occultes de la famille: sorcellerie, « susto $^{6}{ }$, diable ou Satan. Michel n'ayant pas ces connaissances, a eu recours à la religion et à la drogue. Le groupe estime que «quelque chose en lui » regarde le film et semble lui remémorer ce qu'il a vécu avec la Barra Blanca. Malgré le rapprochement des évocations, Michel n'arrive pas à exprimer le traumatisme qu'il a vécu. Il refoule cette expérience et lorsqu'il en parle, c'est avec un certain détachement. Les élaborations sur le traumatisme se modifient habituellement au cours du processus thérapeutique, ce qui n'est pas le cas pour Michel. Une thérapie individuelle pourrait éventuellement lui permettre de faire ces élaborations.

À la quatrième rencontre, la famille est de nouveau au complet. Avant l'entrevue, le groupe discute du «susto», de la sorcellerie et des pratiques magiques qui sont présentes à différents niveaux dans cette famille. Le plan de l'entrevue est d'abord de boire le «maté» ensemble, ensuite de s'informer de la prescription puis de travailler dans le sens de ne pas faire de Michel la «victime» et le «faible» de la famille. L'essentiel de l'entretien se résume à travers les rêves que madame raconte.

\section{Extrait 2}

TP: Reprenons la genèse des difficultés. Ça part des grandsparents de Madame (la thérapeute principale fait une énumération de tout ce qui est arrivé depuis ces grands-parents.... jusqu'à l'histoire de la «Barra Blanca»)

Mère: Je veux raconter un rêve. C'est la fête de Serge et ma mère s'approche de moi. Je veux la rejoindre, elle ne répond pas... Michel apparaît très laid, il est vert avec des cheveux longs. Je demande à ma 
mère pourquoi il est comme cela. Et je ne sais pas. Il y avait longtemps que je n'avais pas rêvé à elle. Et d'habitude, elle est bien.

CT4 : La mère morte vient donner des solutions. Elle et Michel ne sont pas bien...

CT6: Les morts viennent donner des solutions en Amérique Latine

CT5 : Il y a quelque chose de spécial dans la maison actuellement

CT6: En Amérique latine, on peut penser au «limpiar ${ }^{7} »$ avec un cochon d'Inde, avec un breuvage, à une purge...

Mère: Ici on ne trouve pas des connaissances dans ce domaine

CT5 : Ici on cherche ensemble

TP : En conclusion : il faut être attentif aux morts. Ce n'est pas toi Michel qui es le problème mais tu le dénonces et dans ce sens tu es spécial. Comment faire pour te purifier? Faire de toi un homme nouveau. Et comment trouver pour vous madame, quelque chose pour le repos de l'âme de votre mère, et faire purifier l'appartement

Michel: Je vais aller aux bains flottants près de chez moi

Serge: Je sais où tu pourrais aller.

Comme le rêve selon Freud, est la voie royale de l'inconscient, son élaboration par madame à travers le récit de l'Extrait 2 nous fournit un matériel dense qui constitue un mécanisme de projection massive du sentiment et ressentiment éprouvé à l'égard de Michel. Ce rêve nous révèle par sa richesse, la grande culpabilité que vit madame envers son fils, la lourdeur de ce fils qu'elle ne comprend pas et qui lui fait peur. Son besoin de purification de même que celui de son fils Michel font surface, besoin approuvé par les autres membres de la famille présents à la rencontre. Il s'agit d'une pratique déjà expérimentée puisqu'un prêtre est déjà venu purifier l'appartement et que Serge semble connaître les lieux où se pratique ce travail de purification. Le choix du thérapeute principal de suivre une seule piste malgré la multiplicité de ces dernières résulte du fait que c'est celle-là qui a le plus rejoint, à ce moment là, tous les membres de la famille.

À la dernière rencontre, Michel affirme être beaucoup mieux et s'être trouvé du travail. Du coup, sa vie s'est réorganisée entre travail, vie de famille et loisirs. Du coup également, ses interactions avec sa famille se sont améliorées et une distance relationnelle mère-fils a particulièrement pris place et permis à chacun de commencer à déployer sa vie dans des sphères autonomes. Il demeure difficile pour Madame de «lâcher prise » et de laisser aller Michel. Un bilan de l'intervention est 
alors fait où Michel dit avoir beaucoup apprécié être rencontré avec sa famille de façon à ce que les problèmes y soient abordés dans un contexte groupal. L'absence de «conseils» proprement dits à la famille semble toutefois leur avoir manqué, évoquant ici des modes d'aide auxquels ils sont plus familiers.

\section{Analyse}

Lorsque le couple immigre au Québec, madame et monsieur sont confrontés au stress migratoire et aux angoisses des étrangers dans un nouveau pays. Ils ont quitté des personnes importantes, un environnement social familier et connu, une culture bien maîtrisée. Les accouchements de madame sont une nouvelle source de stress, cette expérience étant reconnue comme l'une des situations majeures de fragilisation en pays d'accueil (Bibeau et al., 1992). La mère n'ayant pas été «portée » par son environnement proche à ce moment crucial, n'a pu «porter» ses fils à travers leur enfance ${ }^{8}$ (Winnicott, 1975). Celle de Michel s'est donc déroulée dans un climat difficile d'adaptation et d'intégration de ses parents vivant des deuils et des ajustements à un nouveau pays.

Quant à Michel, sa première migration en Uruguay lui fait perdre son réseau d'amis d'enfance. Son intégration à l'école s'avère particulièrement difficile du fait que lui et son frère ne parlent pas l'espagnol. Les deux frères ont alors à se battre pour se faire respecter car ils sont humiliés et pointés du doigt comme des étrangers. C'est à cette époque que Michel connaît également des difficultés relationnelles avec son père qu'il juge trop sévère à son égard. De retour au Québec, Michel doit de nouveau s'ajuster. Ce troisième changement de lieu de résidence le perturbe beaucoup et il perd confiance en lui à la suite des échecs sur le plan scolaire. Michel éprouve ainsi des difficultés d'affiliation à son groupe d'appartenance. Quel(s) est (sont) son (ses) groupe(s) ? De quels éléments sont-ils constitués ? Quelle est son identité ?

Le divorce de ses parents le bouleverse également. Sa famille connaît alors de grandes pertes matérielles et Michel en est affecté. Il connaît la privation par la faim, la soif et se voit abandonné par son père. Il se laisse aller dans la consommation d'alcool et de drogue pour combler le vide et le manque et affronte la mort lorsqu'il fait la rencontre de la «Barra Blanca». Finalement l'arrivée de Motus provoque beaucoup de frictions faisant de Michel quelqu'un de vulnérable et de plus en plus fragile. 


\section{Plan psychodynamique ou étiologie moderne des difficultés de Michel}

L'état dépressif de la mère avant et après la naissance de Michel n'a pas favorisé le lien avec son enfant qui a réagi en étant un enfant sage, qui ne posait aucun problème. Sa mère était une «mère morte» selon l'expression de Green (1983), c'est-à-dire qu'elle était vivante, mais morte psychiquement aux yeux de Michel. Cette «mort» ayant été déclenchée par les déceptions qui ont provoqué des blessures narcissiques (grossesse peut-être non désirée, humiliation, mésententes, pertes, etc). Michel aurait alors possiblement développé un syndrome d'abandon qui s'est traduit par ce bébé très calme, donc aussi «mort» psychiquement. Cela constitue certes une hypothèse plausible. La mère avait dès les moments de vie de Michel, instauré un lien «mortifère» avec lui, basé sur ses angoisses et ses émotions. Ce qui a inévitablement engendré une certaine peur chez Michel et l'a conduit à s'accrocher à sa mère. De ce lien a résulté une relation symbiotique où Michel s'est trouvé sous l'emprise de sa mère «morte». La mère de Michel ne peut donc nous parler du vécu de sa grossesse que sous l'image de la dépression, ce qui n'est qu'une répétition du message voilé auquel Michel a dû faire face. Enfant, il a dû tenter d'en trouver le sens et en être profondément troublé.

La présence de Motus, considéré comme «un intrus », vient briser la symbiose et la remettre en question par l'exigence d'une différenciation fils-mère. Il en ressort de l'irritation et de la colère que Michel manifeste particulièrement à l'égard de sa mère parce que représentant la zone protégée et intouchable, zone que Berger (1986) désigne de «noyau symbiotique». Au regard des événements vécus dans cette famille et dans la perspective de Green (1983), Motus correspondrait à celui qui vient «guérir» la «mère morte» qui alors s'éveille, s'anime et vit. Michel en est alors abasourdi et se rend compte qu'il est en train de la perdre; toujours selon Green, la mère de Michel «l'abandonne» pour investir dans Motus. Michel a alors beaucoup de difficultés à se détacher de sa mère qui n'est plus morte, il est envahi par l'affect de reviviscence et sa réaction est de démontrer que lui non plus n'est plus «mort». D'où la colère manifeste et les difficultés qu'il rencontre.

Michel reste soumis à l'image idéalisée du père, poursuivant un rêve qu'il n'a jamais pu réaliser au cours de sa formation identitaire. Ce père, comme modèle, est une énigme pour lui car il ne lui a jamais accordé amour et approbation. Pour Michel, la foi mormon représente ce lien essentiel avec le père, elle est un point d'ancrage, un repère dans la recherche d'un père à qui il puisse s'identifier. Nous pouvons 
également interpréter ses références au Che comme un jalon d'identification à ce modèle culturel masculin recherché.

Michel est un enfant qui joue aussi la fonction de lien au sein de sa famille, «il assure à la fois la conjonction et la disjonction des individus, et sa fonction est à la fois celle de conteneur des membres du groupe et de filtre des échanges entre ces membres » (Berger, 1986, 165). Comme le Che a cherché à réunir l'Amérique Latine, Michel a cherché à réunir sa famille.

En résumé, résultant de la multiplicité des ruptures, Michel éprouve de la difficulté à s'investir dans de nouvelles relations par crainte de pertes renouvelées, il y préfère le repli narcissique et la régression dans une position infantile et dépendante. Ainsi peut-on déceler chez lui une angoisse d'abandon de nature dépressive provenant du sentiment nostalgique d'avoir souffert d'une perte irréparable en relation avec la mère dans la prime enfance. L'intolérance à cette angoisse peut se manifester par l'incapacité de distinguer la réalité externe et interne, le bon et le mauvais objet, les bonnes et les mauvaises parties du soi. Elle pourrait aller jusqu'à une angoisse de morcellement de type paranoïde et pré-psychotique décelée par la difficulté à rassembler les parties du moi fragmenté, la méfiance de soi et des autres; facteurs empêchant «d'établir des relations d'objets et de trouver auprès de ces objets le soutien et le plaisir» qui pourraient neutraliser cette angoisse (Klein, 1968, 126).

\section{Plan culturel ou étiologie traditionnelle des difficultés de Michel}

Les prénoms que portent Serge et Michel montrent le désir des parents de s'intégrer à la société québécoise et de laisser derrière eux leur culture. Les différents déplacements de la famille semblent également démontrer une certaine mise à distance des normes essentielles des différentes cultures qui les (les membres) constituent et leur peu d'ancrage culturel.

Michel semble tiraillé entre les exigences de la culture de son père, de sa mère et celles de son pays natal. Les différents systèmes de pensées, les contextes culturels de chacun sont difficiles à saisir pour lui. Il revendique son appartenance québécoise alors que ses autres appartenances sont manifestes. Nous pouvons parler ici de perte d'enveloppe culturelle. Ondong-Essalt $(1998,39)$ réfère à cette notion lorsqu'il dit «émigrer c'est quitter ou perdre l'enveloppe des lieux, des sons notamment la langue maternelle, des odeurs, des goûts, bref des sensations de toutes sortes qui constituent les premières empreintes sur lesquelles s'est établi le codage du fonctionnement psychique et des enveloppes de 
sens ». Chez Michel, elle réfère aux pertes d'identification culturelle résultant des multiples changements de lieux au cours de sa vie, changements qui ne lui ont pas permis de s'imprégner et de baigner suffisamment dans une culture spécifique pour que ses constituants deviennent partie de lui-même.

Michel aurait pu se constituer une matrice culturelle métissée du fait de ses passages successifs dans des univers culturels différents. L'entreprise se révèle toutefois coûteuse sur le plan psychique et certains, comme Michel, entrent dans une répétition mortifère des traumatismes vécus. Tout comme le souligne Moro $(1998,88)$ «la recherche de son identité propre et la construction d'assises narcissiques "sécures" implique un travail d'élaboration sur sa place dans la filiation, sur la représentation de sa famille et de son parcours et in fine une reconstruction patiente de l'estime de soi dans un processus souple et syncrétique». Chez Michel toutefois, cette construction identitaire n'a pu se faire en raison du trop grand nombre de changements dans sa vie et en peu de temps et de la fragilité de son groupe familial.

La famille semble aussi clivée ${ }^{9}$ au niveau des pratiques religieuses, Michel est mormon, la mère est protestante, tous deux chrétiens alors que Serge est très ouvert aux connaissances et pratiques ésotériques et semble maîtriser certaines connaissances et pratiques du monde invisible.

Comme nous l'avons entrevu lors du besoin de purification de madame et de son fils Michel, les problèmes de croyances se traduisent par des pratiques magiques très actives au sein de cette famille, surtout à travers la sorcellerie de la grand-mère de madame et les pratiques du père qui possède une collection de livres sur la magie.

Le bombardement sémantique fait par les co-thérapeutes au regard de leurs cultures a permis de donner à tous une autre vision et compréhension du problème de Michel et a conduit la mère à dérouler l'histoire de la sorcellerie présente dans sa famille. Le recours à ces étiologies traditionnelles a contribué à libérer le discours du patient et de la famille.

Nous sentons toutefois la panique dans laquelle la famille vit par rapport au monde invisible. Tout le monde a peur et chacun cherche à se protéger comme il peut. La mère a l'expérience du domaine de la sorcellerie pour l'avoir vu pratiquée dans sa famille. Les deux fils se sentent vulnérables et tentent de trouver une protection, ils sont alors «exposés ${ }^{10}$ », livrés à eux-mêmes. L'aîné arrive à s'en sortir, il va même assez loin, c'est-à-dire jusqu'à «parler avec les esprits ». Le cadet quant 
à lui, a encore des difficultés à trouver la voie qui lui convient et sa recherche s'oriente vers la pratique de la religion. Le groupe, comme contenant, aide la famille à déposer cette peur suscitée par la reviviscence de pratiques occultes malfaisantes.

\section{Conclusion}

Le transfert positif de Michel et de sa famille envers le groupe des thérapeutes s'est traduit par la compréhension de leurs problèmes. Petit à petit, dans la sécurité d'une relation transférentielle, Michel a confiance au groupe. L'espace et la place que le groupe lui offre lui permet d'exprimer ce qu'il ressent, il parle ouvertement et fait apparaître ses sentiments. Il est aussi amené à entendre l'expression des sentiments des membres de sa famille à son égard.

Nous constatons qu'il y a eu complémentarisme dans le travail fait auprès de cette famille, utilisation à la fois des éclairages psychanalytique et anthropologique. La dynamique de cette famille et de ses membres a ainsi pu être mise à jour par un travail d'explicitation de la vie psychique des personnes et de leurs particularités culturelles. L'articulation psychisme-culture a pu se faire.

Quel est l'avenir de cette approche ethnopsychiatrique dans des sociétés de plus en plus métissées? Dans les métropoles des pays développés, les migrants, fuyant la faim, les persécutions politiques ou des conflits familiaux, se retrouvent depuis nombre d'années livrés, en cas de désordre, à la médecine occidentale. Lorsque des problèmes apparaissent dans ces familles, les intervenants, habitués à leur propre façon de dire et de faire, leur apportent des réponses inadaptées voire déstabilisantes. Or, pour les migrants issus de sociétés non occidentales, il existe déjà des modèles de prise en charge dans leur culture. La consultation dans une clinique ethnopsychiatrique comme la nôtre valide ces modèles et permet à ces migrants d'être entendus psychologiquement, symboliquement et culturellement. Pour cela, il apparaît pertinent que les consultations soient organisées en équipe multidisciplinaire et multiculturelle incluant thérapeutes, médiateurs et intervenants sociaux, pouvant favoriser un «portage» plus approprié des situations.

\section{Notes}

1. Les noms des personnes de cette famille ainsi que les différents pays où elles ont vécu, sauf le Québec, sont fictifs. Ceci dans un souci de préserver l'anonymat des individus.

2. Les mormons sont des chrétiens non catholiques et non protestants. Ils ont été d'une certaine manière à l'écart de la pensée religieuse. Ils ne 
connaissent pas la Bible et ont élaboré leur propre théologie qui comprend 13 Articles de foi rédigé par Joseph Smith le fondateur, en 1842. Celui-ci reçut une autorité «venue d'en haut» et fut habilité par des révélations précises à rétablir l'Église de Jésus Christ sur terre. Leur activité missionnaire est très importante, elle est accomplie sur une période de deux ans, renouvelable par des jeunes gens dont la plupart sont âgés de 19 à 25 ans. Ils catéchisent à leur frais, leurs moyens de subsistance pendant ces deux années provenant de leurs économies personnelles, de l'aide familiale et de l'assistance de la paroisse (Bitton, 1989).

3. Dans une approche ethnopsychiatrique comme la nôtre, elle consiste en une des recommandations thérapeutiques faites par le TP. Elle permet d'établir de nouvelles interactions entre l'individu et les membres de sa famille. Il peut ainsi être question de ramener un objet qui servira à faire la transition, à établir des liens entre les personnes, les lieux et qui pourra faire émerger certains souvenirs précis se rapportant à la difficulté vécue.

4. Il s'agit de la légende du prince Thésée qui réussit à tuer le Minotaure et à sortir du labyrinthe grâce à la pelote de fil que la princesse Ariane lui avait donnée et qu'il accrocha à l'entrée du labyrinthe. Il la dévida derrière lui pour avoir un fil conducteur lui permettant de retrouver son chemin.

5. Le maté ou la Djerba est une tisane très connue en Amérique du Sud. Ce sont des feuilles séchées, réduites presque en poudre. Il a des propriétés stimulantes, tonifiantes et digestives, il est consommé comme le café à la différence qu'il se prend souvent en groupe pour socialiser. Il se boit à la paille à tour de rôle dans un même pot.

6. Maladie attribuée au départ de l'âme hors du corps suite à une frayeur. Les symptômes peuvent apparaître n'importe quand, même des années après l'événement effrayant. Elle peut se présenter sous forme de trouble dépressif majeur, sous forme de stress post-traumatique ou de troubles somatoformes. Le susto peut également entraîner la mort (American Psychiatric Association, 1996).

7. En Amérique Latine, certaines personnes, lorsqu'elles rencontrent des difficultés, peuvent recourir à un traitement, se faire nettoyer (purifier) par un rituel où un cochon d'Inde est utilisé. En Amazonie, sur la côte, on utilise ce qu'on nomme au Pérou le «ayahuasca » qui veut dire : liane des morts. Utilisée en breuvage, elle est hallucinogène et purgative.

8. Winnicot parle de «holding» (manière dont l'enfant est porté), de «handling» (manière dont l'enfant est traité, manipulé) et d' «object presenting » (mode de présentation de l'objet) lors des premiers stades du développement émotionnel et mental du nourrisson et de l'enfant. Lorsque l'enfant est «porté» et «traité» de façon satisfaisante 
(principalement par la mère, figure précurseure du miroir et composante objectale de l'environnement), sa réponse aux divers efforts de son environnement pourra être adéquate.

9. Il s'agit ici de la coexistence de pratiques magico-religieuses différentes, voire opposées au sein d'une même famille et qui distinguent ses membres.

10. Enfants exposés réfère à une notion à la fois théorique et clinique, opératoire en pratique lorsqu'il s'agit d'enfants de migrants. Emprunté à la mythologie, il s'agit d'enfants abandonnés à un milieu hostile. «Ces enfants courent un risque, celui du passage d'un monde culturel à un autre; en quelque sorte le danger qui les menace est celui de mort culturelle» (Moro 1989, 69).

\section{Références}

American Psychiatric Association, 1996, DSM-IV Manuel diagnostique et statistique des troubles mentaux, Annexe 1, Paris, Masson.

Bitton, D., 1989, Les Mormons, Éditions du Cerf, Paris.

Berger, M., 1986, Entretiens familiaux et champ transitionnel, PUF, Paris.

Bibeau, G., Chan-Yip, A. M., Lock, M., Rousseau, C., Sterlin, C., Fleury, H., 1992, La santé mentale et ses visages: un Québec pluriethnique au quotidien, Gaëtan Morin éditeur, Boucherville.

COHEN-EMERIQue, M., 1990, Le modèle individualiste du sujet : écran à la compréhension des personnes issues des sociétés non occidentales, Cahiers de sociologie économique et culturelle, 13, juin.

CoRBEIL, L., 1997, La consultation d'ethnopsychiatrie : une pratique de la différence, Intervention, 105.

DevereuX, G., 1972, Ethnopsychanalyse complémentariste, Flammarion, Paris.

Devereux, G., 1970, Essais d'ethnopsychanalyse générale, Gallimard, Paris.

Dumont, L., 1978, La conception moderne de l'individu, Esprit, 2, février.

GreEn, A., 1983, Narcissisme de vie et narcissisme de mort, Les Éditions de Minuit, Paris.

KIRMAYER, L. J., 1989, Psychotherapy and the cultural concept of the person, Santé, Culture, Health, 6, 3.

KiRMAYER, L., Minas, H., 2000, The future of cultural psychiatry: an international perspective, Canadian Journal of Psychiatry, 45, 438-446. 
KLEIN, M., 1968, Envie et gratitude et autres essais, Gallimard, Paris.

Kleiman, A. M., 1977, Depression, somatization and the new cross-cultural psychiatry, Social Science and Medicine, 11, 3-10.

Laplantine, F., 1988, L'ethnopsychiatrie, Presses Universitaires de France, Paris.

Moro, M. R., 1989, D'où viennent ces enfants si étranges ? Logiques de l'exposition dans la psychopathologie des enfants de migrants, L'enfant exposé, Nouvelle Revue d'ethnopsychiatrie, 12.

Moro, M. R., 1992, Principes théoriques et méthodologiques de l'ethnopsychiatrie, Santé mentale au Québec, XVII, 2, 71-98.

Moro, M. R., 1998, Psychothérapie transculturelle des enfants de migrants, Dunod, Paris.

Nathan, T., 1986, La folie des autres: traité d'ethnopsychiatrie clinique, Dunod, Paris.

Nathan, T., 1988, Le sperme du diable: éléments d'ethnopsychiatrie, Presses universitaires de France, Paris.

Nathan, T., 1994, L'influence qui guérit, Éditions Odile Jacob, Paris.

Ondong-Essalt, E., Flot, C., 1998, L'ethnopsychiatrie communautaire, Revue internationale d'études transculturelles et d'ethnopsychanalyse clinique, 1.

StReIT, U., 1996, Les migrants : Adaptation de la théorie psychanalytique dans un contexte interculturel, in Doucet, P. et Reid, W., éds., La psychothérapie psychanalytique, Gaëtan Morin éditeur, Boucherville.

TshiseKedi-Kalanga, M. R, 2002, Santé mentale et clientèle immigrante: l'approche ethnopsychiatrique, mémoire de maitrise, École de Service Social, Université de Montréal.

WinNicotT, R. W., 1975, Jeu et réalité, Gallimard, Paris.

\section{ABSTRACT}

\section{Analysis of a clinical intervention according to instruments inspired by the ethnopsychiatric approach elaborated by Devereux and Nathan}

This article analyses a clinical intervention that was carried out at the ethnopsychiatric unit of the Jean-Talon hospital in Montreal. The authors first present an overview of the central concepts of an 
ethnopsychiatric approach elaborated in France by Nathan in response to mental health problems experienced by immigrants. The authors' intervention is in line with this ethnopsychiatric approach currently being developed in Quebec. The clinical situation is then presented followed by a description of the intervention carried out by the ethnopsychiatric unit. Finally, the authors conclude with an analysis of the situation from various perspectives: psychosocial, psychodynamic (modern etiology) and cultural (traditional etiology).

\section{RESUMEN}

\section{Análisis de una intervención clínica en relación con los instrumentos elaborados por el enfoque etnopsiquiátrico de Devereux y Nathan.}

Este artículo analiza una intervención clínica efectuada en el Módulo de etnopsiquiatría del Hospital Jean-Talon de Montreal. Los autores presentan primero un recordatorio de ciertas nociones centrales de un enfoque etnopsiquiátrico desarrollado en Francia por Nathan en respuesta a los problemas de salud mental vividos por los migrantes. La presentación de su intervención se inscribe en la trayectoria de este enfoque, el cual está en proceso de elaboración y experimentación en Québec. Después, se presenta la situación clínica seguida de la intervención efectuada por el Módulo de etnopsiquiatría. Por último, los autores terminan con un análisis de la situación en el plano psicosocial, psicodinámico (etiología moderna) y cultural (etiología tradicional).

\section{RESUMO}

\section{Análise de uma intervenção clínica em função das ferramentas elaboradas pela abordagem etnopsiquiátrica de Devereux e Nathan}

Este artigo analisa uma intervenção clínica efetuada no Módulo de etnopsiquiatria do Hospital Jean-Talon de Montreal. Os autores relembram, primeiramente, algumas noções centrais de uma abordagem etnopsiquiátrica desenvolvida na França por Nathan, em resposta aos problemas de saúde mental vivenciados pelos imigrantes. A apresentação de sua intervenção inscreve-se na trajetória desta abordagem, que está em processo de elaboração e de experimentação no Quebec. A situação clínica é apresentada, seguida da intervenção efetuada pelo Módulo de etnopsiquiatria. Enfim, os autores concluem com uma análise da situação no plano psicossocial, psicodinâmico (etiologia moderna) e cultural (etiologia tradicional). 


\section{Graphique \\ Représentation du dispositif-groupe pour la famille de Michel}

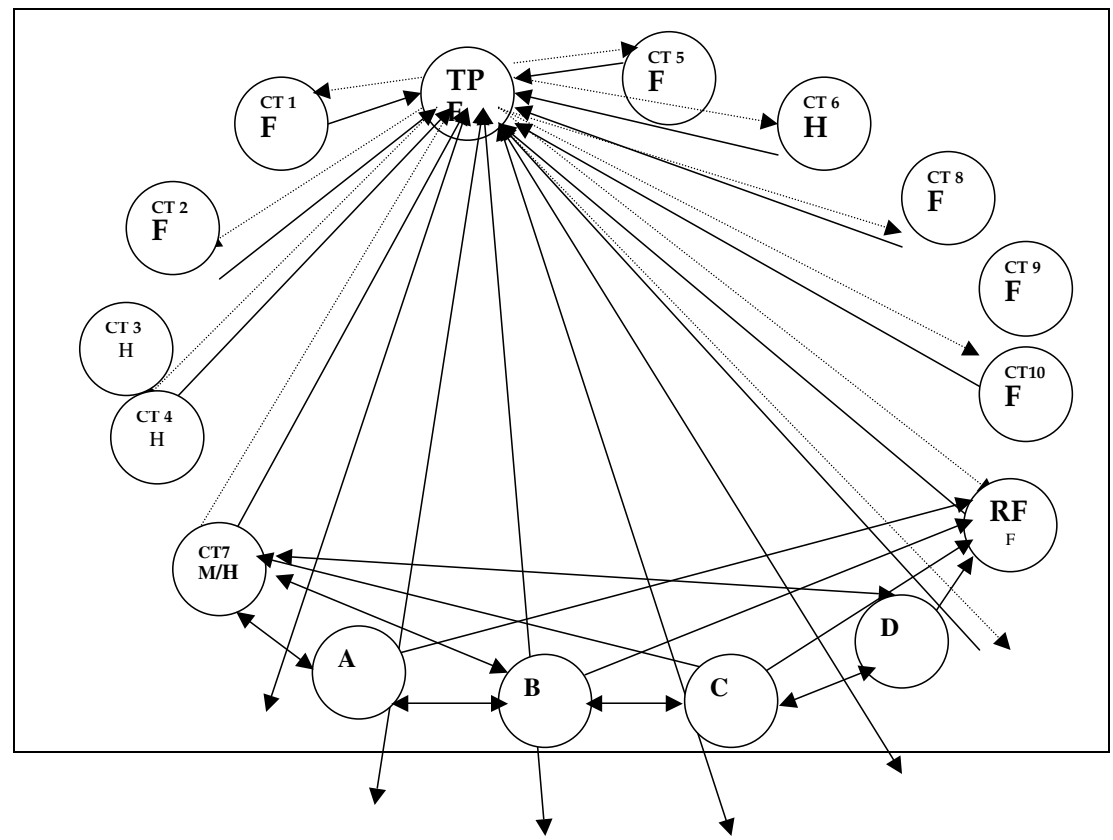

\section{LÉGENDE}

TP: Thérapeute principale

CT : Co-thérapeute

$\mathrm{M}$ : Médiateur

$\mathrm{H}:$ Homme

F: Femme

$\mathrm{R}$ : Référante

A : Motus, Chilien, conjoint de Madame

$\mathrm{B}$ : Madame, Argentine, mère de $\mathrm{C}$ et de $\mathrm{D}$

C: Serge, fils aîné

D : Michel, fils cadet, patient désigné

$\mathrm{C}$ et $\mathrm{D}$ : nés au Québec de père uruguayen
TP: Québécoise, criminologue

CT 1: Haïtienne, travailleuse sociale

CT 2: Québécoise, psychologue

CT 3 : Haïtien, médecin

CT 4: Marocain, psychiatre

CT 5: Québécoise, travailleuse sociale

CT 6: Libanais, intervenant social

CT 7: Québéco-Péruvien, médiateur, psychologue

CT 8: Chilienne, psychologue

CT 9: Française, Psychologue

CT10: Congolaise, stagiaire en service social

RF : Française, travailleuse sociale

$\longrightarrow$ : Les flèches pleines indiquent le sens de l'échange.

......... : Les flèches pointillées indiquent la réponse du TP à une intervention pertinente qui peut faire avancer le processus en cours. 